\title{
Optimal hybridisation and management of PV/batteries hybrid systems in residential distribution networks
}

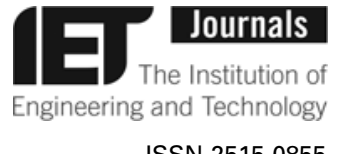

ISSN 2515-0855 doi: 10.1049/oap-cired.2017.1155 www.ietdl.org

\author{
Harun Turker $\bowtie$, Patrick Favre-Perrod \\ University of Applied Sciences, School of Engineering and Architecture of Fribourg, \\ Fribourg, Switzerland \\ $凶$ E-mail: harun.turker.1984@gmail.com
}

\begin{abstract}
This study presents a decentralised off-line strategy for optimal hybridisation and management of photovoltaic (PV)/batteries hybrid systems in residential distribution grids. The aim is to study the voltage plan of the network without/ with this process for different penetration rates of PVs and also comparing with the initial grid without PV. The tool has been tested by using a real Swiss distribution network and real data of houses electricity consumption. In addition, a voltage assessment methodology has been proposed. Lot of study cases has been performed and the results show that the voltage plan of the grid is greatly improved, thanks to the tool proposed. For quantify the results, one performance index, which assesses the quality of the network voltage has been introduced.
\end{abstract}

\section{Introduction}

Developed countries promote the residential photovoltaic (PV) energy production systems, which are thriving. Due to their dependence on weather conditions, the renewable energy sources including PV have a stochastic production, which makes their integration into the electric grid difficult, particularly due to the voltage problems.

The literature on the solar energy production (SEP) control with an energy storage system is widespread but the strategies are principally centralised and require information and communication technologies (ICT) for real time implementation. To this, works with aims to improve the penetration rates of PV systems [1], minimise lines losses of the grid [2], peak shaving [3], voltage control [4] by assessing at the same time the economic aspects [5] were proposed. To a lesser extent, on-line energy management strategies (EMS) $[6,7]$ and decentralised algorithms are also available [8].

In this paper, a different approach is proposed in order to solve the integration of PVs systems into the electric grid. A generic tool, i.e. an offline EMS, composed of two algorithms is proposed. In first step, the EMS defines the constant maximum power injection into the grid by the PV/batteries hybrid system during all the day. For that, an off-line linear programming (LP) optimisation algorithm has been developed where the daily SEP is known. In the second step, the minimum optimal battery size which allows to obtain a full energy efficiency has been defined with a heuristic process. In this context, the energy efficiency represents the quantity of solar energy produced from the PV panels injected into the grid. At last, to assess the quality of the network voltage, a methodology proposed is used.

The proposed EMS is generic and he has the capacity to work for any quantity of SEP (low power systems in residential area or high power systems as power plants). The tool proposed has a strong feature because it is weakly linked to new ICT deployments and it is decentralised. Indeed, it can be used now for existing power grids without additional infrastructure. The proposed tool is destined to industrials, R\&D engineering and distribution system operators (DSO). Depending up on the users, he is versatile. It aims to help in design step of a hybrid system. It will allow to choose the optimal battery size and to make technical-economic analysis. During operation, it is useful to define the daily energy production plan of a PV/battery hybrid system by determining the constant maximum power.

\section{Methodology}

This part presents the voltage assessment methodology, which is composed in three main steps (Fig. 1).

\subsection{Step 1: grid load}

This step consists to affect a daily load profile (DLP) at each housing in the low-voltage (LV) network (without PV production).

Substep 1.1: The disparity concerning the size of houses has been taken into account. As such, the houses have a size varying from three rooms to six rooms [9]. A pseudo-random (uniform distribution) size select process is performed for each housing. Probabilities have been set as following: $20 \%$ for three rooms house, $20 \%$ for four rooms house, $30 \%$ for five rooms house and $30 \%$ for six rooms house. Given that the LV network used as a use case is residential, the probability of 'large' houses select has been favoured.

Substep 1.2: This substep consists to select randomly a DLP at each housing in database of 1000 real DLPs. For that, are used the databases of real DLPs for each house size (from three to six rooms) in summer season $[10,11]$. Same DLP has not been chosen for several housing.

Substep 1.3: The databases of real DLPs provide active powers. Therefore, a reactive power for each housing is generated thanks to a random select of $\tan \varphi$ between 0 and 0.5 [12].

Substep 1.4: The randomly location process of each house allows mixing the different sizes positions in the LV network.

\subsection{Step 2: PV production integration}

In this step, the stochastic production of PV is integrated in the grid without control (and so, without storage system).

Substep 2.1: A theoretical penetration rate (PR) is chosen by the user. The PR is defined from the daily energy consumed by the grid. During assessment work (part III), PR has been varied from $10 \%$ to full.

Substep 2.2: Depending the PR, the number of PV systems is defined by using the initial daily solar energy production (DSEP). A smart method was applied to modify the nominal power of each PV system integrated into the grid. If the number of PV system in the network is one, initial DSEP is used. Otherwise, for each PV 


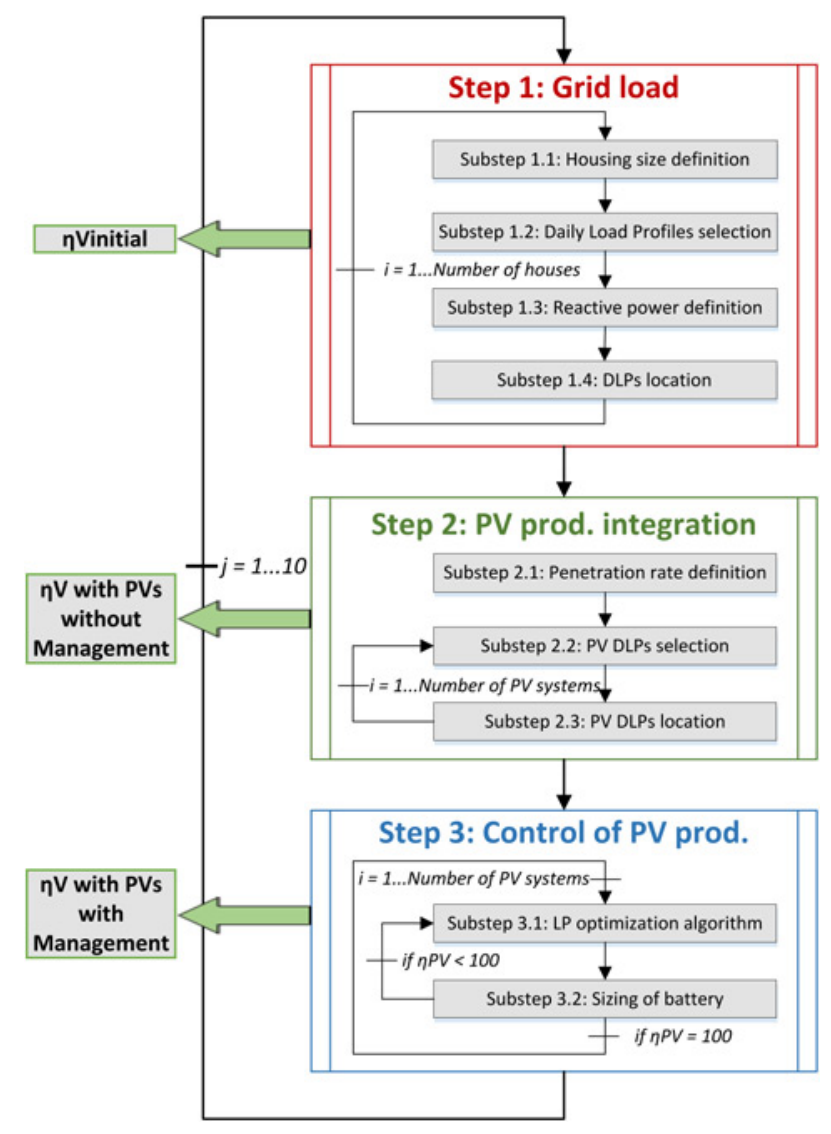

Fig. 1 Synoptic of the voltage assessment methodology

system, (1) is used to define randomly a multiplication factor which is applied at the initial DSEP. Fig. 2 shows the shape of the DSEP; initial DSEP has a peak production equals to $3 \mathrm{~kW}$ around

$$
\operatorname{MF}[0.3 ; 1.7]=((1.7-0.3) \times A[0 ; 1])+0.3
$$

The variability of the solar production is performed through the randomly selection (between 0 and 1 ) of the variable $A$. The shape of the initial DSEP has been considered as generic and typical for a PV system without energy storage equipment. This shape is preserved and used in all simulations. The min and max values of the solar production have been chosen in line with the nominal powers of the systems available on the market.

Substep 2.3: Each PV system is randomly located into the network.

\subsection{Step 3: control of PV production with storage}

The last step of the voltage assessment methodology consists to manage the PV/battery hybrid system.

Substep 3.1: LP optimisation algorithm.

An off-line LP optimisation algorithm has been developed for define the maximum constant power injection into the grid during all the day within the constraints (Fig. 3). The EMS is off-line because the DSEP is known by the algorithm. An off-line algorithm was chosen because it allows to assess the theoretical optimum achievable in a real-time strategy.

The optimisation problem formulation is as follows:

In this system, the state and control variables are represented by the battery state-of-charge (SOC) and the power at inject into the grid, respectively (2)

$$
P_{\text {Grid }}(t)=P_{\mathrm{PV}}(t)+P_{\text {Battery }}(t) \quad \forall t \in[0,1440]
$$

The limits related to the battery pack formed the inequality constraints (3) and (4) available $\forall t \in[0,1440]$

$$
\begin{aligned}
& P_{\text {Batt-Disch.-Max }} \leq P_{\text {Battery }}(t) \leq P_{\text {Batt-Charge-Max }} \\
& \operatorname{SOC}_{\text {Min }} \leq \mathrm{SOC}(t) \leq \mathrm{SOC}_{\text {Max }}
\end{aligned}
$$

There are two equality constraints. The first: the power injected into the grid must be constant during all the day (5). The second: the battery SOC at the end of the day must be equal to his value of the beginning of the day (6)

$$
\begin{gathered}
P_{\text {Grid }}(t)=P_{\text {Grid }}(t-1) \quad \forall t \in[1,1440] \\
\operatorname{SOC}_{\text {Final }}\left(t_{\mathrm{f}}\right)=\operatorname{SOC}_{\text {Initial }}\left(t_{\mathrm{i}}\right) \text { with } t_{\mathrm{i}}=0, t_{\mathrm{f}}=1440
\end{gathered}
$$

The cost criterion is defined by (7).

$$
E_{\text {Grid }}=\int_{t_{\mathrm{i}}=0}^{t_{\mathrm{f}}=1440} P_{\mathrm{PV}}(t)+P_{\text {Battery }}(t) \mathrm{d} t
$$

The system under study and the constraints are represented by linear equations. That is why the LP was chosen. The total number of sampling time steps is 1440 for the simulation of one day; $1 \mathrm{~min}$ is the best compromise between precision and calculation time.

The Linprog tool provided by Matlab has been used for solve the optimisation problem. Interior-point method has been employed.

The Coulomb metric method has been used for the dynamic monitoring of the battery SOC [11].

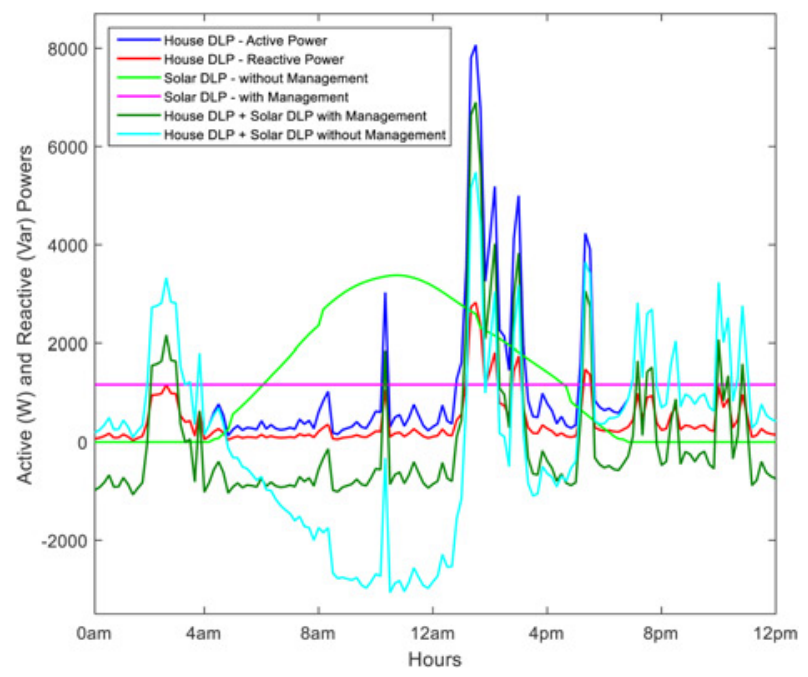

Fig. 2 Application of Linear Programming (LP) optimization algorithm

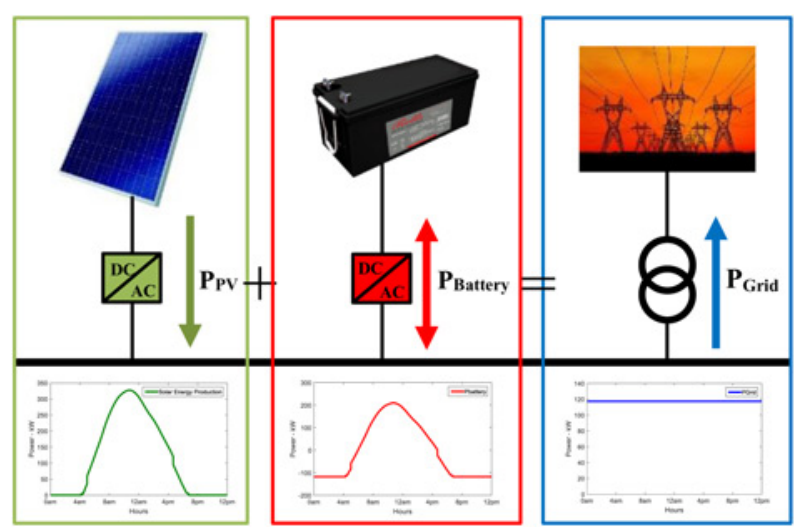

Fig. 3 Synoptic of a Grid-connected PV/Battery hybrid system 
Substep 3.2: Optimal sizing of battery.

In this Substep, the minimum optimal battery size which allows us to obtain a full energy efficiency has been defined with a heuristic process. First, a value is fixed arbitrarily and then, this one is increased (or decrease) following the result of the efficiency obtained. In this context, the energy efficiency represents the quantity of solar energy produced from the PV panels injected into the grid. The heuristic process and so, the research of the optimal battery size, is performed with a step equal to $100 \mathrm{Wh}$ which is a good compromise between precision and calculation time.

Fig. 2 illustrates an example related to step 3.

\subsection{Voltage assessment methodology}

Information 1: The methodology ensures that the cases treated in the step 3 are the same selected in the previous step. For each housing, this concerns: the selection of the size (number of rooms), the DLP, $\tan \varphi$, the position in the grid, the PV DLP, the PV location into the network etc.

Information 2: For each PR study (more information is given in the next part), the global process is repeated 10 times; which is sufficient since the criterion introduced do not change beyond this. Indeed, the convergence is achieved at 10 iterations.

\section{Simulation results}

\subsection{Methodology application}

The PR of PVs has been varied from $10 \%$ to full and for each PR, 10 simulations have been performed. Fig. 4 shows for all cases the number of houses selected depending their size. Full PR corresponds to one PV system for each housing. In this case, the energy produced by all PVs systems during the day represents around $130 \%$ of the daily energy consumed by the grid. In the same way (i.e. for all cases) Fig. 5 illustrates the total energy consumed by the network during all the day (without PV integration). Table 1 presents the number of PV installations. In a logical way, more the PR is high and more the diversity in the number of PV installations is high. Fig. 6 shows the position into the network of the PV systems for a PR equal to $20 \%$.

It is found that the randomness sought thanks to the methodology is well represented.

\subsection{Voltage plan of the network}

A real residential distribution network made available by Groupe-E (DSO in Switzerland) has been used. It is an urban electric grid

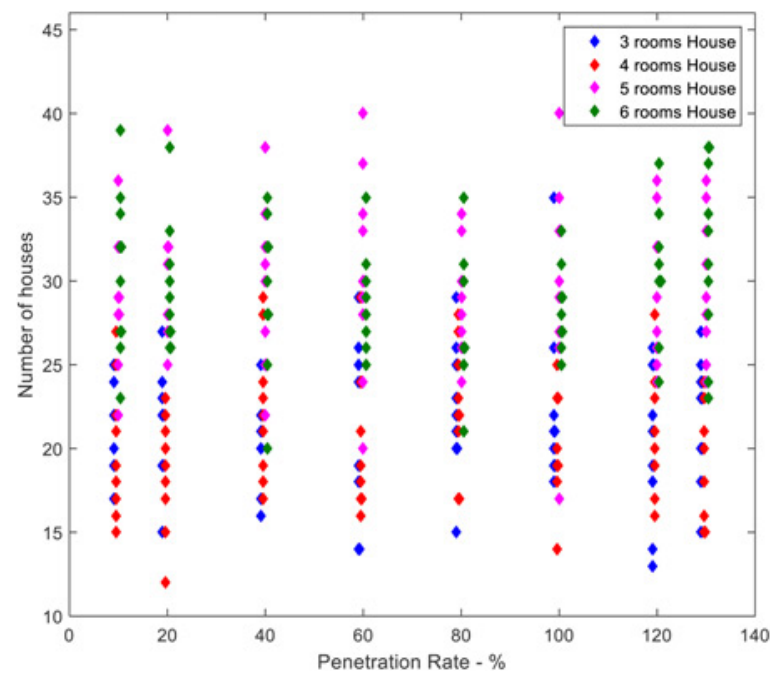

Fig. 4 House size selection

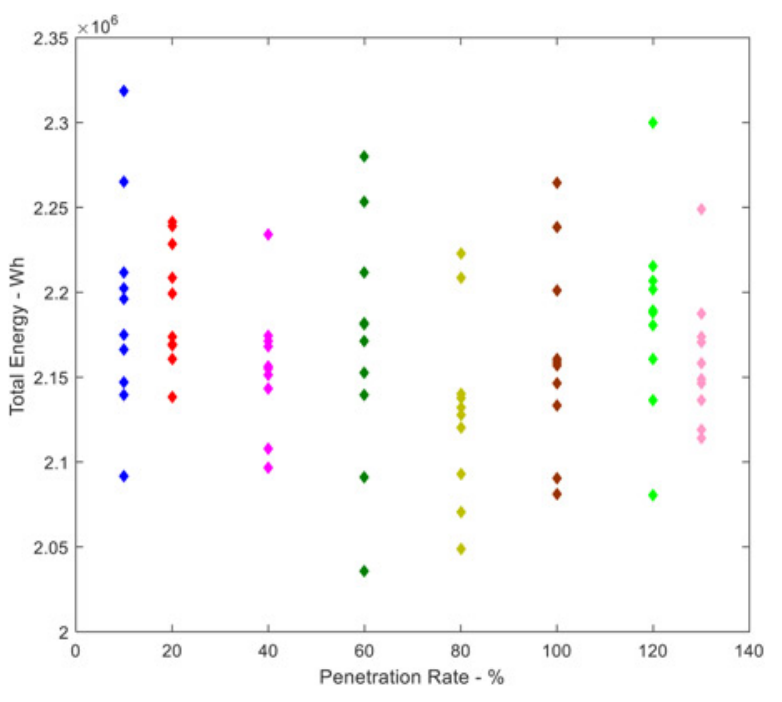

Fig. 5 Total energy consumed by the network

Table 1 Number of PV installations

\begin{tabular}{lcccccccccc}
\hline Simus & 1 & 2 & 3 & 4 & 5 & 6 & 7 & 8 & 9 & 10 \\
\hline PR10 & 8 & 8 & 8 & 8 & 8 & 8 & 8 & 8 & 8 & 8 \\
PR20 & 16 & 16 & 16 & 16 & 16 & 15 & 16 & 16 & 16 & 16 \\
PR40 & 31 & 31 & 31 & 31 & 32 & 30 & 31 & 31 & 31 & 31 \\
PR60 & 47 & 47 & 45 & 49 & 50 & 46 & 47 & 48 & 44 & 47 \\
PR80 & 62 & 59 & 64 & 60 & 61 & 62 & 61 & 62 & 62 & 64 \\
PR100 & 78 & 80 & 78 & 78 & 81 & 75 & 77 & 76 & 78 & 82 \\
PR120 & 96 & 100 & 95 & 96 & 95 & 96 & 90 & 94 & 93 & 95 \\
full & & & \multicolumn{7}{c}{101} &
\end{tabular}

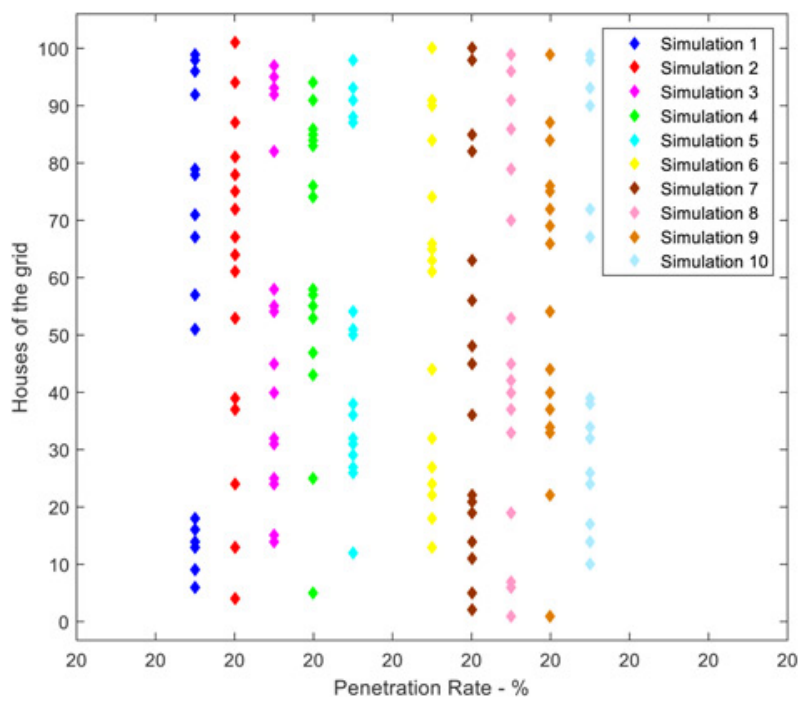

Fig. 6 Position into the grid of the PV systems

(underground power cables) which powers 101 housing (Fig. 7). Neplan software has been used to do the load flow (LF) analysis.

For each PR (8 in total), 10 simulations have been performed in first without PV and then with the same conditions but with PV integration without control and in last with management of the PV systems.

For that, 24,240 houses DLPs have been used and 240 simulations of daily LF have been performed. Each daily LF is composed to 144 LF because the houses DLPs and solar DLPs used have a sampling time equal to $10 \mathrm{~min}$.

Figs. 8 and 9 show the voltage plan for PRs equals to $40 \%$ and full, respectively. For each of the three cases, Figs. 8 and 9 illustrate the average daily voltage profile obtained from 10 


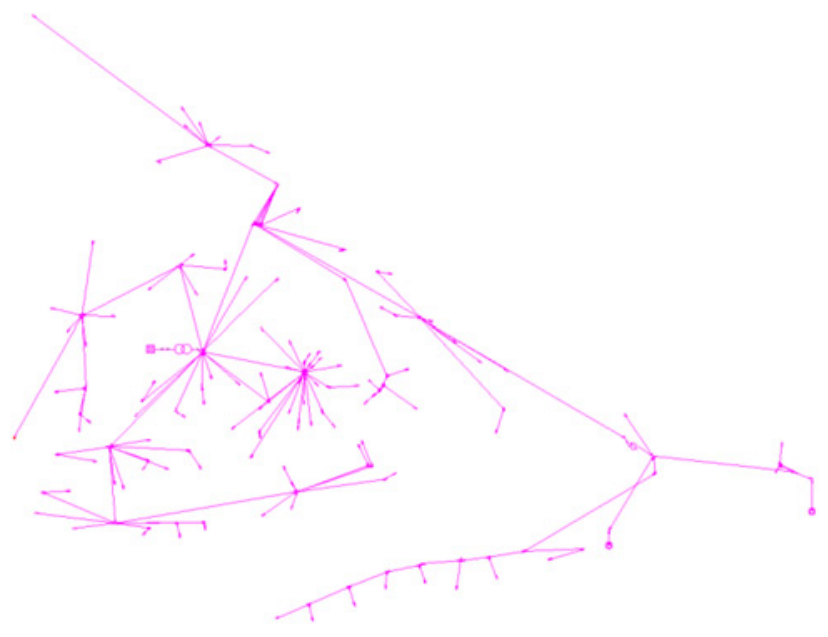

Fig. 7 Real residential distribution network in Fribourg, Switzerland

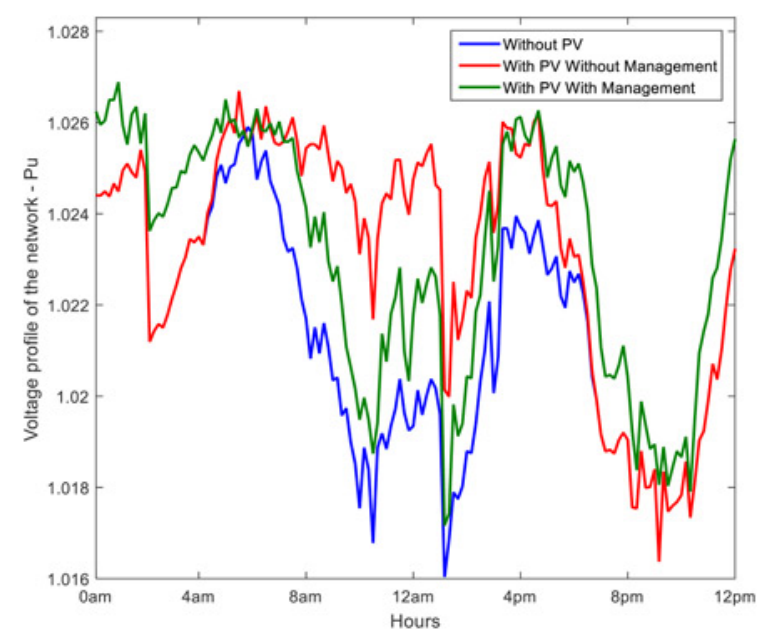

Fig. 8 Voltage plan of the network $-P R=40 \%$

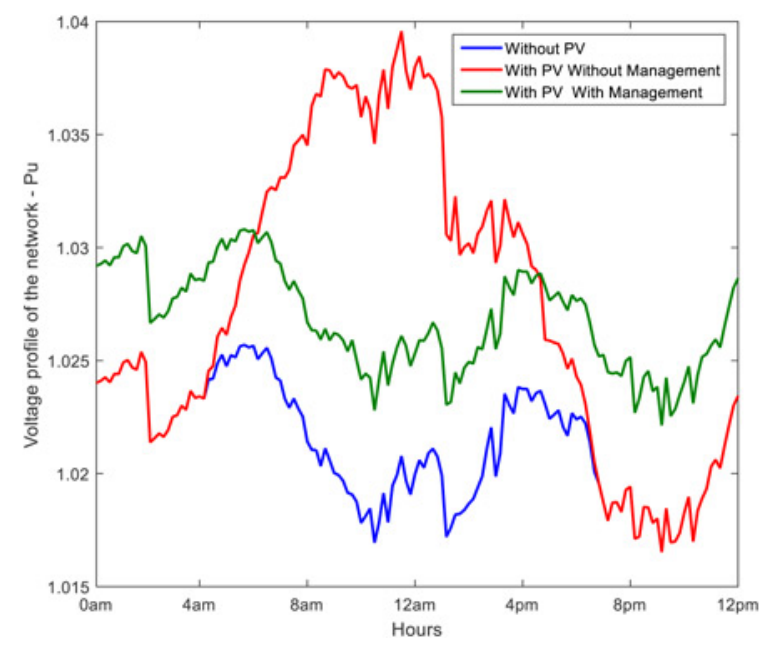

Fig. 9 Voltage plan of the network $-P R=$ full

simulations. For each simulation, the voltage profile of the network is obtained by calculated the mean from all the nodes.

The electricity distributor agrees to respect the standard NF-EN-50160 which imposes to supply customers with a $\sim 400 \mathrm{~V}$ voltage between $\pm 10 \%$. Out of these limits, it is considered that the voltage plan is no longer respected.

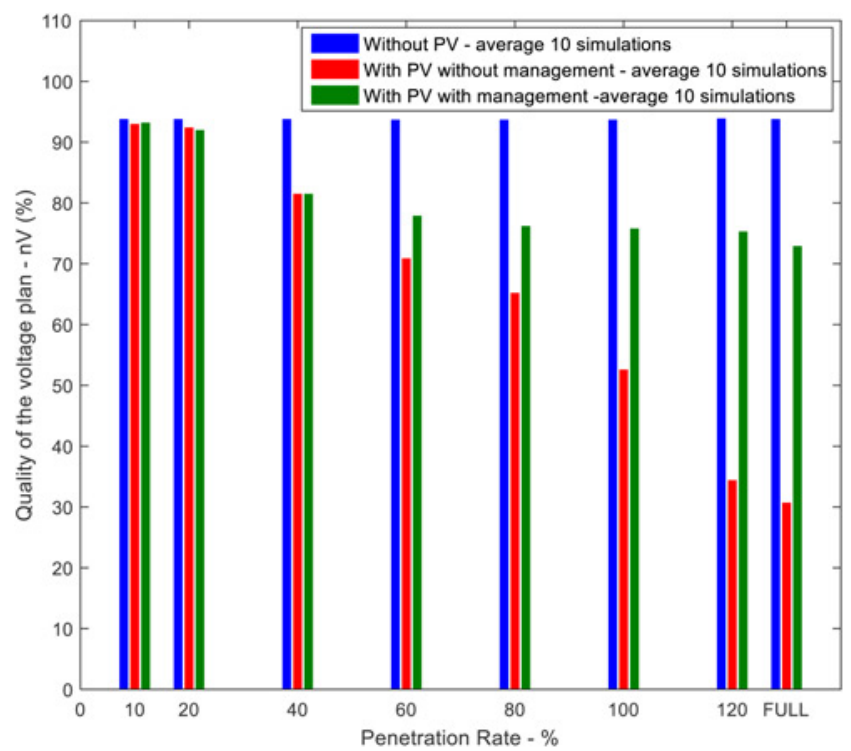

Fig. 10 Quality of the voltage plan of the network

For all simulations [10 simulations $\times 3$ cases (without PV, and with $\mathrm{PV}$ with/without control) $\times 8 \mathrm{PRs}$ ], daily voltage profiles of each of the 165 nodes of the grid have been analysed. Among these many cases, all meet the standard. Indeed, as in many developed countries, the residential distribution networks are currently oversized.

One of the major problems with PV integration is the overvoltages. In this way and to study this, the standard has been stressed and new limits have been tested: $\sim 400 \mathrm{~V}$ between $-10 \%$ and $+3.5 \%$. A criterion, $\eta \mathrm{V}$, has been introduced. This one represents the quality of the global voltage plan [13]. The index $\eta \mathrm{V}$ counts for each of the eight penetration rates and for each of the three cases (without PV, and with PV with/without control), the number of cases among the 1.650 where the standard is not respect during a duration greater than $10 \mathrm{~min}$. To standardise the index $\eta \mathrm{V}$, for each of the three cases, one has at most one-time per day where the standard is not respected for each node in each of the 10 simulations. Fig. 10 shows the results of $\eta \mathrm{V}$ for all the cases.

\section{Conclusions}

In this paper, a decentralised off-line strategy for optimal hybridisation and management of $\mathrm{PV} / \mathrm{batteries}$ hybrid systems in residential distribution grids has been proposed. Lot of study cases have been performed by using a real Swiss distribution network and real data of houses electricity consumption. A proposed voltage assessment methodology has been used. A performance index has been introduced to assess the quality of the grid voltage.

Based on statistic performed from the results, a logical way, it is shown that uncoordinated integration of PV systems without storage system degrades the voltage plan of the residential grid. The higher the PR is the higher the impact.

With management of the hybrid systems, which consists to inject a constant power during all the day, it is observed that the quality of the voltage is greatly improved. Indeed, the major degradation of the voltage plan caused by the uncoordinated massive integration of PV systems is canceled. A strong finding is made, thanks to the tool proposed; the voltage plan of the grid reaches (practically) the same quality with management regardless the PR of the PVs.

The tool proposed in this paper is off-line because the solar production is known. The real PV production is not known in advance and it is a difficult field. This will be treated in the next paper for a better implementation of the solution proposed here. 


\section{Acknowledgments}

The authors would like to thank EOS Holding/SCCER FURIES for the financial support.

\section{References}

1 Tant, J., Geth, F., Six, D., et al.: 'Multiobjective battery storage to improve PV integration in residential distribution grids', IEEE Trans. Sustain. Energy, 2013, 4, (1), pp. 182-191

2 Teng, J. H., Luan, S. W., Lee, D. J., et al.: 'Optimal charging/discharging scheduling of battery storage systems for distribution systems interconnected with sizeable PV generation systems', IEEE Trans. Power Syst., 2013, 28, (2), pp. $1425-1433$

3 Yang, Y., Li, H., Aichhorn, A., et al.: 'Sizing strategy of distributed battery storage system with high penetration of photovoltaic for voltage regulation and peak load shaving', IEEE Trans. Smart Grid, 2014, 5, (2), pp. 982-991

4 Kabir, M. N. Mishra, Y, Ledwich, G, et al. "Coordinated control of grid-connected photovoltaic reactive power and battery energy storage systems to improve the voltage profile of a residential distribution feeder', IEEE Trans. Ind. Inf., 2014, 10, (2), pp. 967-977

5 von Appen, J., Stetz, T., Braun, M., et al.: 'Local voltage control strategies for PV storage systems in distribution grids', IEEE Trans. Smart Grid, 2014, 5, (2), pp. 1002-1009
6 Perez, E., Beltran, H., Aparicio, N., et al.: 'Predictive power control for PV plants with energy storage', IEEE Trans. Sustain. Energy, 2013, 4, (2), pp. 482-490

7 Cavey, M. V., Helsen, L., Tant, J., et al.: 'Real-time control for services provided by battery energy storage systems in a residential low voltage grid with a large amount of PV'. 22nd Int. Conf., Exhibition on Electricity Distribution (CIRED 2013), 2013

8 Marra, F., Yang, G., Træholt, C., et al.: 'A decentralized storage strategy for residential feeders with photovoltaics', IEEE Trans. Smart Grid, 2014, 5, (2), pp. $974-981$

9 Turker, H., Bacha, S., Chatroux, D., et al.: 'Low-voltage transformer loss-of-life assessments for a high penetration of plug-in hybrid electric vehicles (PHEVs)', IEEE Trans. Power Deliv., 2012, 27, (3), pp. 1323-1331

10 Turker, H., Bacha, S., Hably, A.: 'Rule-based charging of plug-in electric vehicles (PEVs): impacts on the aging rate of low-voltage transformers', IEEE Trans. Power Deliv., 2014, 29, (3), pp. 1012-1019

11 Turker, H., Bacha, S., Chatroux, D., et al.: 'Modelling of system components for vehicle-to-grid (V2G) and vehicle-to-home (V2H) applications with plug-in hybrid electric vehicles (PHEVs)', Innovative Smart Grid Technologies (ISGT), 2012 IEEE PES, 16-20 January 2012

12 Turker, H., Florescu, A., Bacha, S., et al.: 'Voltage profile and excess subscription assessments indexes based on random selection of real daily loads profiles (DLPs) on residential electric grid areas for a high penetration of plug-in hybrid electric vehicles (PHEVs)'. Vehicle Power and Propulsion Conf. (VPPC), 2011 IEEE, September 2011

13 Turker, H., Hably, A., Bacha, S.: 'Smart charging of plug-in hybrid electric vehicles (PHEVs) on the residential electric grid regarding the voltage plan'. Energy Conversion Congress and Exposition (ECCE), 2013 IEEE, September 2013 